\title{
PROJECTIVELY NORMAL ADJUNCTION SURFACES
}

\author{
MARCO ANDREATTA AND EDOARDO BALLICO
}

(Communicated by Louis J. Ratliff, Jr.)

\begin{abstract}
On a projective surface $S$ polarized by a very ample line bundle $L$ one can consider the adjoint bundles $\left(K_{S} \otimes L\right)^{\otimes n}=\boldsymbol{L}^{\otimes n}$ and the adjunction mappings associated to them. Suppose these mappings are embeddings (it is well known when this is the case: see [So-VdV]). We prove that these embeddings are projectively normal for $n \geq 2$ and we describe some counterexamples for $n=1$. For $n \geq 2$ we show that the ideals of the image of $S$ are generated by quadrics.
\end{abstract}

Let $S^{\prime}$ be a projective surface polarized by a very ample line bundle $L^{\prime}$ and let $\boldsymbol{L}^{\prime}=K_{S^{\prime}}+L^{\prime}$ be the adjoint bundle. Let $r: S^{\prime} \longrightarrow S$ be the contraction of all the rational curves $P \subset S^{\prime}$, such that $L^{\prime} \cdot P=-K_{S^{\prime}}^{\prime} \cdot P=1$, to a finite number of smooth points in a new surface $S$. Of course the map associated to $\Gamma\left(\boldsymbol{L}^{\prime}\right)$ will factor through $r$, and $\boldsymbol{L}^{\prime}=r^{*}\left(K_{S}+L\right)=r^{*}(\boldsymbol{L})$, where $L$ is an ample line bundle on $S$ such that $L=r_{*}\left(L^{\prime}\right)$. In [So-VdV] it is proved that if $\left(S^{\prime}, L^{\prime}\right)$ is not in a well-known list of polarized pairs then the map associated to $\Gamma(\boldsymbol{L})$ is an embedding (we just mention that this paper concludes the study of the very ampleness of the bundle $L$, using the technique of Reider, which was a very old question going back to Castelnuovo and Enriques (see also [Se1]).

It is natural at this point to ask for the projective normality of this embedding (we will say $\boldsymbol{L}$ is projectively normal). If there is a smooth hyperelliptic curve $C$ in the linear system $|L|$, then, by the Noether theorem and the adjunction formula, $\boldsymbol{L}$ is not projectively normal (precisely $\boldsymbol{L}$ is not 1 - and 2 -normal); but even if there is no such element in $|L|$ we will show an example for which the projective normality of $\boldsymbol{L}$ fails.

However we prove that $\boldsymbol{L}^{\otimes n}$ is projectively normal for all $n \geq 2$, and that the ideal of the image of the surface $S$ through the map associated at $\Gamma\left(L^{\otimes n}\right)$ is generated by quadrics.

Moreover, if $R(\boldsymbol{L}, S)=: \bigoplus_{m} H^{0}\left(O_{S}(m \boldsymbol{L})\right)=\bigoplus_{m} R_{m}$ is the graded ring associated to $L$, then $r(L, S)$, the maximal degree of the elements of $R(L, S)$ which appears in a minimal system of homogeneous generator, is less than or equal to two.

Received by the editors January 31, 1990 and, in revised form, June 20, 1990.

1980 Mathematics Subject Classification (1985 Revision). Primary 14J99, 14H99, 14 N05.

Key words and phrases. Adjoint bundle, adjunction mapping, projective normality. 
The proofs rely on the theory of the Koszul cohomology developed by Mark L. Green $[\mathrm{Gr}]$.

\section{NOTATION AND PRELIMINARIES}

1.1. In this paper we consider projective surfaces $S$, which means that $S$ is a smooth, irreducible, projective scheme of dimension 2, defined over the field of complex numbers.

If $D$ is a divisor on a surface $S$ we will use the following notation:

- $O_{S}(D)=$ : the invertible sheaf associated to $D$,

- $h^{i} O_{S}(D)=: \operatorname{dim} H^{i}\left(O_{S}(D)\right)$,

- $\chi\left(O_{S}(D)\right)=: \sum_{i=0,1,2}(-1)^{i} h^{i} O_{S}(D)$,

- $K_{S}=:$ the canonical divisor on $S$,

- $g(D)=: \frac{1}{2}\left(D \cdot D+D \cdot K_{S}\right)+1$, the arithmetic genus of $D$,

- $p_{g}(S)=: \operatorname{dim} H^{0}\left(O_{S}\right)$, the geometric genus of $S$,

- $q(S)=: \operatorname{dim} H^{1}\left(O_{S}\right)$, the irregularity of $S$,

- $k(S)=: \operatorname{tr} \operatorname{deg}\left(\bigoplus_{n=0}^{\infty} H^{0}\left(S, O_{S}\left(n K_{S}\right)\right)\right)-1$, the Kodaira dimension of $S$.

We do not distinguish notationally between a divisor and its associated line bundle.

1.2. According to what we have said in the introduction and to what we are going to prove, from now on we will consider a line bundle $L$ on $S$ which is at least nef and big and such that $L=K_{S}+L$ is spanned by global sections and big.

1.3. We can associate to $L$ a graded ring

$$
R(\boldsymbol{L}, S)=: \bigoplus_{m} H^{0}\left(O_{S}\left(\boldsymbol{L}^{\otimes m}\right)\right)=\bigoplus_{m} R_{m},
$$

and we define the $\boldsymbol{L}$-dimension of $S$ by

$$
k(S, \boldsymbol{L}):=\operatorname{tr} \operatorname{deg} R(\boldsymbol{L}, S)-1 .
$$

From our assumption 1.2 we have that $k(S, L)=2$ and, by a theorem of Mumford [Mu], that $R(L, S)$ is finitely generated.

Finally $r(\boldsymbol{L}, S)$ will denote the maximal degree of the elements of $R(\boldsymbol{L}, S)$ which appears in a minimal system of homogeneous generators.

1.4. If we call $\varphi_{n \boldsymbol{L}}: S \longrightarrow \mathbf{P}^{N}$ the map associated to $\boldsymbol{L}^{\otimes n}:=\boldsymbol{H}$ for $n \geq 1$, we have the following

Definition. $\boldsymbol{H}$ is said to be projectively normal if for every $\rho \geq 0$ the maps

$$
H^{0}\left(\mathbf{P}^{N}, O(\rho)\right) \cong S^{\rho} H^{0}(S, \boldsymbol{H}) \longrightarrow H^{0}\left(S, \boldsymbol{H}^{\rho}\right)
$$

are surjective. 
1.4.1. Remark. In the case $\rho=0$ the maps are surjective for all $n \geq 1$; in the case $\rho=1$ they are surjective if $\boldsymbol{H}$ is very ample.

2. THE KosZUL COHOMOLOGY AND THE PROJECTIVE NORMALITY OF $\boldsymbol{L}^{\otimes n}$

As mentioned in the introduction, we will obtain our results via the theory of the Koszul cohomology as developed by Mark L. Green in [Gr], which is applied to our particular case, that is, on a projective surface $S$ for the line bundle $L=K_{S}+L$ with $L$ nef and big. We constantly refer directly to [Gr].

2.1. We briefly recall that in the notation of 1.3 , that is, with $R_{m}=H^{0}\left(S, L^{\otimes m}\right)$, we can construct the Koszul complex

$$
\cdots \rightarrow \Lambda^{p+1} R_{1} \otimes R_{q-1}-\stackrel{d_{p+1, q-1}}{\longrightarrow} \Lambda^{p} R_{1} \otimes R_{q} \stackrel{d_{p, q}}{\rightarrow} \Lambda^{p-1} R_{1} \otimes R_{q+1} \longrightarrow \cdots
$$

and the Koszul cohomology groups are defined by

$$
\mathscr{K}_{p, q}(S, \boldsymbol{L}):=\operatorname{Ker} d_{p, q} / \operatorname{Im} d_{p, q} .
$$

We now prove the following

2.2. Proposition. Let $S, L$, and $\boldsymbol{L}$ be as in 1.2. Then $\mathscr{K}_{p, q}(S, \boldsymbol{L})=0$ for all $q \geq 4$ and $p \leq p_{g}(S)-q(S)+g(L)-3$ and for $q=3$ and $p \leq g(L)-q(S)-3$.

Proof. By the Kodaira vanishing theorem and the Serre duality we have

$$
H^{1}\left(S, L^{\otimes p}\right)=0 \text { for } p \neq 0 ;
$$

hence for $q \geq 3$, we are in the hypothesis of the "duality theorem," that is, the theorem 2.c.6 in [Gr].

This tells us that

$$
\left(\mathscr{K}_{p, q}(S, \boldsymbol{L})\right)^{*}=\mathscr{K}_{r-2-p, 3-q}\left(S, K_{S}, \boldsymbol{L}\right) \quad \text { with } r=h^{0}(\boldsymbol{L})-1 .
$$

On the other hand the "vanishing theorem," that is, the Theorem 3.a.1 in [Gr], asserts that

$$
\mathscr{K}_{r-2-p, 3-q}\left(S, K_{S}, L\right)=0 \text { if } h^{0}\left(S, K_{S}-(q-3) L\right) \leq r-2-p .
$$

If $q \geq 4$ then $h^{0}\left(S, K_{S}-(q-3) L\right)=0$, by the hypothesis, and therefore we are asking for

$$
p \leq r-2=h^{0}(\boldsymbol{L})-3=p_{g}(S)-q(S)+g(L)-3 .
$$

If $q=3$ then $h^{0}\left(S, K_{S}-(q-3) L\right)=p_{g}(S)$, and we are asking for

$$
p \leq g(L)-q(S)-3 .
$$

2.3. Corollary. In the hypothesis of the proposition and if $h^{0}(L) \geq 4$, we have

$$
R_{m}=R_{1} R_{m-1} \text { for all } m \geq 3 \text {. }
$$

Proof. By the construction of the Koszul cohomology and since $\Lambda^{p} R_{1}=0$ for $p<0$, we have that our corollary will follow immediately if we prove that $\mathscr{K}_{0, q}(S, \boldsymbol{L})=0$ for all $q \geq 3$. 
Since, by hypothesis, we can assume that $p_{g}(S)-q(S)+g(L)=h^{0}(\boldsymbol{L}) \geq 3$ we have immediately from the proposition that $\mathscr{K}_{0, q}(S, L)=0$ for all $q \geq 4$.

On the other hand $g(L)-q(S)-3=h^{0}(\boldsymbol{L})-h^{0}\left(K_{S}\right)-3 \geq 0:$ this is true as above if $h^{0}\left(K_{S}\right)=0$ and by the Hopf lemma otherwise (i.e., $h^{0}(\boldsymbol{L})-h^{0}\left(K_{S}\right)=$ $\left.h^{0}\left(K_{S}+L\right)-h^{0}\left(K_{S}\right) \geq h^{0}(L)-1 \geq 3\right)$. This will give $\mathscr{K}_{0, q}(S, L)=0$ for $q=3$.

As a consequence of the corollary we have the following

2.4. Theorem. Let $S$ be a projective surface, and $L$ a nef and big line bundle on $S$ such that $h^{0}(L) \geq 4$ and $\boldsymbol{L}=K_{S}+L$ is spanned by global sections and big.

Then

(a) $r(S, L) \leq 2$,

(b) for every $n \geq 2$, if $\boldsymbol{L}^{\otimes n}$ is very ample then it is projectively normal.

If moreover $h^{0}(\boldsymbol{L}) \geq 4$, then

(c) the ideal of $\varphi_{n L}(S)$ is generated by quadrics.

Proof. (a) and (b) follow immediately from Corollary 2.3. (c) on the other hand follows from $\mathscr{K}_{1, q}(S, L)=0$ for all $q \geq 3$; this is proved as in the case $p=0$ with the hypothesis $h^{0}(\boldsymbol{L}) \geq 4$.

2.5. Remark. As pointed out in the introduction, consider a polarized pair $\left(S^{\prime}, L^{\prime}\right)$ with $L^{\prime}$ very ample and which is neither $\left(\mathbf{P}^{2}, O(e)\right)$ with $e=1$ or 2 , nor a quadric, nor a scroll, nor a conic bundle, nor a Del-Pezzo surface; then $\boldsymbol{L}^{\prime}=K_{S^{\prime}}+L^{\prime}$ is spanned by global sections and big and the map associated to $\boldsymbol{L}^{\prime \otimes n}$ for $n \geq 2$, after contracting the (-1)-lines relative to $L$, is an embedding (see [So-VdV]). By our theorem this embedding is projectively normal and the ideal of the image is generated by quadrics.

\section{THE ADJOINT BUNDLE $L$}

Assuming that $\boldsymbol{L}$ is very ample, it is natural at this point to ask for the projective normality of $\boldsymbol{L}$ itself.

By the previous result this is equivalent at the surjectivity of the map

$$
S^{2} H^{0}(S, \boldsymbol{L}) \longrightarrow H^{0}\left(S, \boldsymbol{L}^{\otimes 2}\right)
$$

(the other being surjective by Corollary 2.3 ).

Unfortunately this map is not surjective in many cases: for instance, as mentioned in the introduction, that is the case if $|L|$ contains a smooth hyperelliptic element, by the adjunction formula, the Noether theorem, and the Kodaira vanishing theorem.

On the other hand the map is not surjective as soon as

$$
\operatorname{dim} H^{0}\left(S, \boldsymbol{L}^{\otimes 2}\right)>\operatorname{dim} S^{2} H^{0}(S, \boldsymbol{L}),
$$


or equivalently

$$
\left(p_{g}(S)-q(s)+g(L)\right)^{2}<p_{g}(S)+3 g(L)-q(S)-2+2 d^{\prime}
$$

with $d^{\prime}=\boldsymbol{L} \cdot \boldsymbol{L}$.

Polarized surfaces $(S, L)$ with $\boldsymbol{L}$ very ample, with no smooth hyperelliptic element in $|L|$ and satisfying this inequality, and therefore not projectively normal relatively to $L$, exist: for instance the abelian surfaces of degree 10 in $\mathbf{P}^{4}$.

Another surface of this type is the following: let $S$ be a minimal bielliptic surface in $\mathbf{P}^{4}$, i.e., $S \subset \mathbf{P}^{4}$ is a minimal surface with $k(S)=0, q(S)=1$, $p_{g}(S)=0$. F. Serrano proved recently the existence of such a surface and that for it we have $L \cdot L=\boldsymbol{L} \cdot \boldsymbol{L}=10$, and $g(L)=6$ [Se2].

\section{FURTHER REMARKS}

In this section we describe one of our initial motivations for the construction of as many projectively normal surfaces $S \subset \mathbf{P}^{N}$ as possible; the reader will find no theorems here. We fix one such surface and we assume moreover that $h^{i}\left(\boldsymbol{O}_{S}(1)\right)=0$ for $i=1,2$ (as in the cases $\left|\boldsymbol{O}_{S}(1)\right|=|2 K+2 L|$, etc.). Calling $H=\boldsymbol{O}_{S}(1)$ we let $d=\operatorname{deg} S=H \cdot H$ and $g=g(H)$.

Now take a general hyperplane $M \subset \mathbf{P}^{N}$ and set $C:=S \cap M$. We have $h^{1}\left(\boldsymbol{O}_{C}(1)\right)=p_{g}$ and $h^{1}\left(\boldsymbol{I}_{C, M}(1)\right)=q ; H^{0}\left(M, \boldsymbol{O}_{M}(t)\right) \rightarrow H^{0}\left(C, \boldsymbol{O}_{C}(t)\right)$ are surjective for every $t \geq 2$ and in particular $C$ has maximal rank. We think that the curves obtained in this way are very interesting.

First of all, using the Euler sequence, we get $h^{1}\left(N_{C / M}\right) \geq p_{g} N-(4 g-2)$. Thus very often $h^{1}\left(N_{C / M}\right)$ is huge; for instance if $|H|=|2 K+2 L|$, this $h^{1}$ is always $\neq 0$ if $p_{g} \geq 8$. The standard degeneration methods to obtain curves of maximal rank [Ba-El 1; $\mathrm{Ba}-\mathrm{El} 2]$ show only the existence of curves, $D$, with maximal rank and with $h^{1}\left(N_{D / M}\right)=0$. Furthermore, by construction, these curves $C$ are in the unknown part of $M_{g}$ which is the union of rational curves.

Now fix an irreducible component $W$ of $\mathrm{Hilb}\left(\mathbf{P}^{N-1}\right)_{\text {red }}, W$ parametrizing (part of the) curves of given degree $d$ and arithmetical genus $g$, and let $W^{\prime} \subset W$ the part parametrizing curve with at most one node as singularities (alternatively: any number of nodes allowed) and with maximal rank. Assume $N \geq 6$. Fix a general point $x \in \mathbf{P}^{N}$ such that $x \notin \operatorname{Sec}(S)$ and also a (general) Lefschetz pencil $A$ with a $\mathbf{P}^{N-2}$ not containing $x$ as axis. By the generality of $M$ and the pencil, we may take $M$ to be an element of the pencil. We assume, since $M=\mathbf{P}^{N-1}$, that $C \in W$ (hence $C \in W^{\prime}$ ). Projecting from $x$ to $M$ we find a pencil of smooth curves in $W$. They are all nondegenerate and with maximal rank, except the one corresponding to the hyperplane of $A$ containing $x$. Thus we get an injective morphism from the affine line to $W^{\prime}$.

Over $\mathbf{C}$ the existence of nonconstant holomorphic maps $\mathbf{C} \rightarrow W^{\prime}$ gives mild restrictions on the complex function theory of $W^{\prime}$. 
To obtain more, we assume from now on that (as in Theorem 2.4) the homogeneous ideal of $S$ is generated by quadrics. Fix a point $\mathbf{P} \in C$. Note that the assumption on the homogeneous ideal of $S$ implies that for every line $D \subset M$ through $P$, length $(D \cap C) \leq 2$. This means exactly that the image $C^{\prime}$ of $C$ under the projection from $P$ into a $\mathbf{P}^{N-2}$, say $M^{\prime}$, is smooth with $C^{\prime} \cong C$ and (under this isomorphism) $\boldsymbol{O}_{C^{\prime}}(1) \cong \boldsymbol{O}_{C}(1) \otimes \boldsymbol{O}_{C}(-P)$. Fix a general line $R \subset M$ with $\mathbf{P} \in R$. Assume $N \geq 7$. For general $R$ we may find such a line $R$ with $\{P\}=\operatorname{Sec}(C) \cap R$ (quasi-transversal intersection at $P$ ). Fix a general $\mathbf{P}^{N-1}$ and consider the family $\left\{C_{y}\right\}_{y \in R}$ of projections of $C$ into $\mathbf{P}^{N-1}$ from $y \in R$ as $y$ varies. For $y \neq P\left(C_{y, \mathbf{P}^{N-1}}\right)$ is projectively equivalent to $(C, M)$ (i.e., under the standard identification of $\mathbf{P}^{N-1}$ and $M$ given by the projection from $y$, $C_{y}$ goes to $C$ ). For $y=P$ by [Ba-El 1, proof of Proposition I.1], we get that $C_{P}$ is the union of $C^{\prime} \subset M^{\prime}$ and a line $E$ linked to $C^{\prime}$ (see [Ba-El 1, picture at p. 4]; $E$ is the image of the plane spanned by $R$ and $T_{P} C$ ). The condition that $S$ and $C$ are scheme-theoretically cut out by quadrics means exactly that in $M$ there are $N-2$ quadrics of $M$ containing $C$ and whose tangent spaces at $P$ have intersection $T_{P} C$. This means that the subspace of $H^{0}\left(M, I_{C, M}(2)\right)$ formed by quadrics which are cones of vertex $P$ has codimension $N-2$ (as small as possible). If (as very often in our situation) $h^{1}\left(\boldsymbol{O}_{C^{\prime}}(2)\right)=0$, this means that the restriction map $H^{0}\left(M^{\prime}, \boldsymbol{O}_{M^{\prime}}(2)\right) \rightarrow H^{0}\left(C^{\prime}, \boldsymbol{O}_{C^{\prime}}(2)\right)$ is surjective; very often it is very easy to check the corresponding surjectivity statement for forms of degree $>2$. Using a Mayer-Vietoris exact sequence we get the surjectivity of $H^{0}\left(M, \boldsymbol{O}_{M}(t)\right) \rightarrow H^{0}\left(C^{\prime} \cup E, \boldsymbol{O}_{C^{\prime} \cup E}(t)\right)$ for every $t \geq 2$.

Thus we find in a suitable $W^{\prime}$ a complete family (parametrized by $\mathbf{P}^{1}$ ). For instance this implies that $W \backslash W^{\prime}$ does not contain the support of an ample divisor.

\section{REFERENCES}

[Ba-El1] E. Ballico and P. Ellia, On degeneration of projective curves, Algebraic Geometry-Open Problems-Ravello, Lecture Notes in Math., vol. 997, Springer-Verlag, New York, 1983, pp. $1-15$.

[Ba-El2] _ (1989), 1-22.

[Gr] M. L. Green, Koszul cohomology and the geometry of projective varieties, J. Differential Geom. 19 (1984), 125-171.

[Mu] D. Mumford, The canonical ring of an algebraic surface, Ann. of Math. (2) 76 (1962), 612-615.

[Se1] F. Serrano, The adjunction mapping and hyperelliptic divisors on a surface, J. Reine Angew. Math. 381 (1987), 90-109.

[Se2] _ Divisors of bielliptic surfaces and embeddings in $\mathbf{P}^{4}$, preprint.

[So-VdV] A. J. Sommese and A. Van de Ven, On the adjunction mapping, Math. Ann. 278 (1987), 593-603.

Dipartimento di Matematica, Universita di Milano, via Saldini 50, Milano, Italia

Dipartimento di Matematica, Università di Trento, 38050 Povo (TN), Italia 